\title{
Appendix 5: Proposers of Motions
}

\begin{tabular}{|c|c|c|c|c|}
\hline No. & References & Date & Formula & Comments \\
\hline 1. & $F d D 3.224$ & $\begin{array}{l}\text { end } 3 \text { rd c. } \\
\text { BCE }\end{array}$ & $\begin{array}{l}{\left[\dot{\varepsilon} \pi \varepsilon \lambda \theta \dot{\omega} \nu \pi 0 \tau i \tau \dot{\alpha} \nu \dot{\varepsilon} \kappa \varkappa \lambda \eta \sigma \sigma^{\prime} \alpha \nu\right]} \\
\text { 'having come upon the assembly' }\end{array}$ & $\begin{array}{l}\text { hieromnamon } \\
\text { from Chios }\end{array}$ \\
\hline 2. & $F d D 2.94$ & 2nd с. BCE & 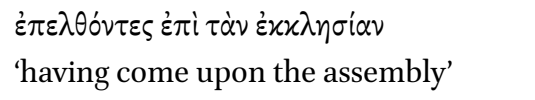 & $\begin{array}{l}\text { ambassadors } \\
\text { from Athens }\end{array}$ \\
\hline 3. & $S G D I 2677$ & 189/8 ВСЕ & $\begin{array}{l}\dot{\varepsilon} \pi i \tau \dot{\alpha} \nu \dot{\varepsilon} \varkappa \varkappa \lambda \eta \sigma i \alpha \nu \dot{\alpha} \nu \alpha \gamma \gamma \varepsilon 1 \lambda \alpha[\nu] \\
\text { 'they proclaimed upon the assembly' }\end{array}$ & theoroi \\
\hline 4. & $F d D 4_{4.427} \mathrm{~B}, \mathrm{I}$ & 189/8 ВСЕ & 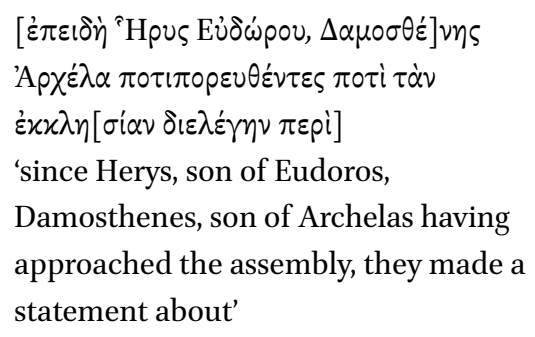 & \\
\hline 5. & $F d D 3.241$ & 166 вСЕ & 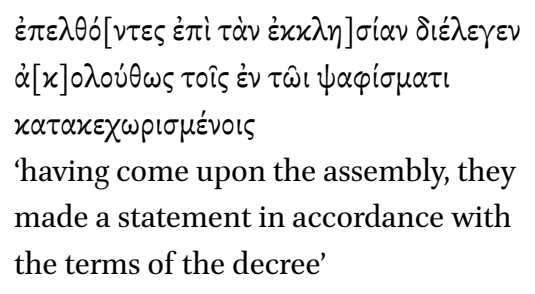 & $\begin{array}{l}\text { ambassadors } \\
\text { from Sardis }\end{array}$ \\
\hline 6. & $F d D_{3.242}$ & 166 вСЕ & 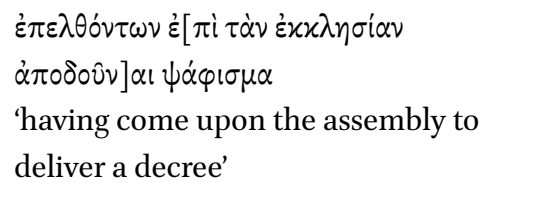 & $\begin{array}{l}\text { ambassadors } \\
\text { from Sardis }\end{array}$ \\
\hline 7. & $\begin{array}{l}\text { Klio } \\
18.1923 .286,213\end{array}$ & 159 ВСЕ & 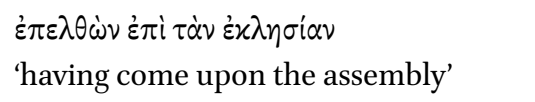 & \\
\hline
\end{tabular}


No. References

Date

Formula

Comments

8. $\quad F d D 1.153$

$146 / 5$ B CE

$\dot{\varepsilon} \pi \varepsilon 1[\delta \dot{\eta}$

छे] $\pi \varepsilon \lambda \theta \dot{\omega} \nu$ غ่ $\pi \dot{\imath}$

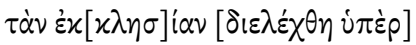

'since ... having come upon the assem-

bly, he made a statement about'

9. $\quad S G D I 2683$

140-100

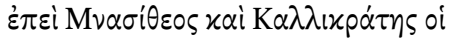

brothers (see

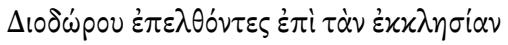

nos. 10-12)

$\delta เ \varepsilon \lambda[\dot{\varepsilon}] \gamma \eta \sigma \alpha \nu$ ن́ $\pi \dot{\varepsilon} \rho$

'since Mnasitheos and Kallikrates,

sons of Diodoros having come upon

the assembly, they made a statement

about'

10. $B C H 68 / 69.1944 /$

134 ? 130 ?

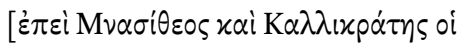

brothers (see $5.105,14$

120 ? ВCE

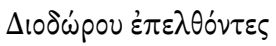

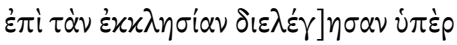
nos. 9 , 11 and

'since Mnasitheos and Kallikrates, sons of Diodoros, having come upon the assembly, they made a statement about'

11. $\quad F d D_{3.118}$

139 BCE

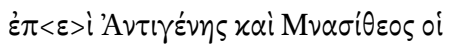

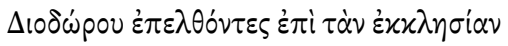

$\delta เ \varepsilon \lambda \varepsilon ́ \gamma \eta \sigma \alpha \nu$ ن́ $\pi \dot{\rho} \rho$

'since Antigenes and Kallikrates, sons of Diodoros, having come upon the assembly, they made a statement about'

12. Klio 17.1921.193.184

139 BCE

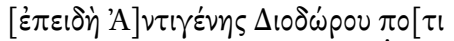

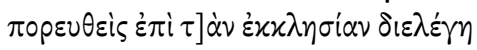
i $\pi \dot{\rho} \rho$

'since Antigenes, son of Diodoros, having approached the assembly, he made a statement about' brothers (see nos. 9, 10 and

12); Antigenes was a bouleutes at that time

12)

brothers (see nos. 9-11); Antigenes was a bouleutes at that time 


\begin{tabular}{|c|c|c|c|c|}
\hline No. & References & Date & Formula & Comments \\
\hline 13. & $\begin{array}{l}\text { ВСН } 68 / 69.1944 / \\
5.103,12\end{array}$ & $\begin{array}{l}\text { 142/1 or } \\
141 / \text { O BCE }\end{array}$ & 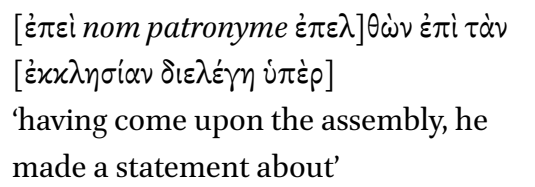 & \\
\hline 14. & $F d D$ 4.171 & $\begin{array}{l}\text { 142/1 or } \\
141 / \text { о BCE }\end{array}$ & 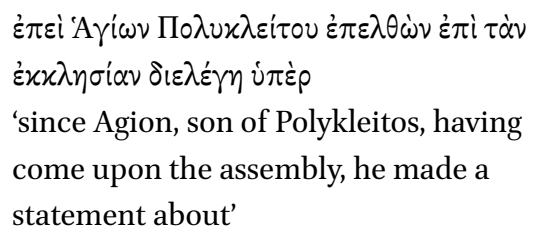 & \\
\hline 15. & $F d D 4.174$ & $\begin{array}{l}\text { 142/1 or } \\
141 / \text { В ВСE }\end{array}$ & 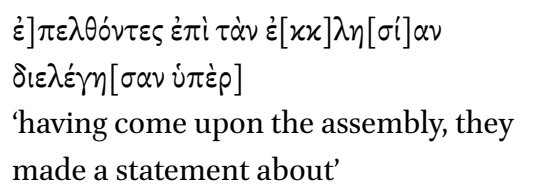 & $\begin{array}{l}\text { ambassadors } \\
\text { from Herakleia }\end{array}$ \\
\hline
\end{tabular}

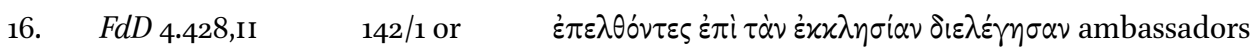
141/O BCE i $\pi \dot{\mathrm{\varepsilon} \rho}$ from Hypata 'having come upon the assembly, they made a statement about'

17. $B C H$

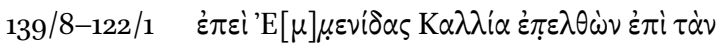

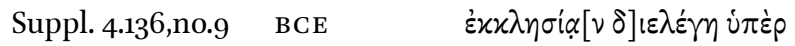
'since Emmenidas, son of Kallias, having come upon the assembly, he made a statement about'

18. $\quad F d D_{3.147}$

139 B CE

$[\dot{\varepsilon}] \pi \varepsilon[i]$ 'A $\gamma \dot{\prime} \omega \nu \mathrm{K}[\lambda] \varepsilon[0] \delta \alpha \dot{\alpha} \mu \nu \mathrm{\varepsilon} \pi \varepsilon \lambda \theta \dot{\omega} \nu$

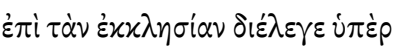
'since Agion, son of Kleodamos, Agion was a bouleutes at having come upon the assembly, he made a statement about'

19. $\quad F d D 6.4$

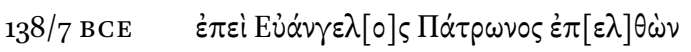

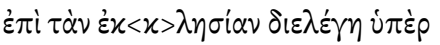
'since Euangelos, son of Patron, having come upon the assembly, he made a statement about' 
No. References

20. $F d D$ 1.261[2]

21. Klio

18.1923.299.224

22. $F d D 4.172$

23. $B C H$ $5^{2.1928 .174[2] ~}$

24. $\quad F d D 4.5^{6}$ and 4.57

91-68 в СЕ

10О-5О ВСЕ$$
x \lambda
$$$$
\text { 'since Emmenidas, son of ... and ... }
$$$$
\text { having come upon the assembly, they }
$$$$
\text { made a statement about' }
$$

\section{Comments}

ambassadors from Hypata $\delta เ \varepsilon \lambda \dot{\varepsilon} \gamma[\eta \sigma] \alpha \nu$ i $\pi \dot{\varepsilon} \rho$ having come upon the assembly, they made a statement about'

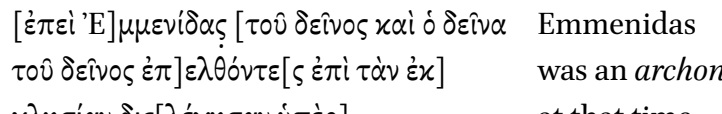

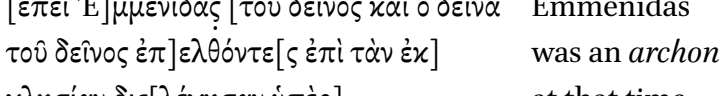
at that time

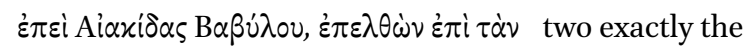

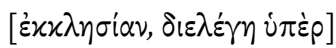
same texts 'since Aiakidas, son of Babulon, having come upon the assembly, he made a statement about'

25. $\quad F d D 1.463$ 55-50 вСе

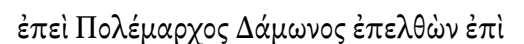
$\tau \dot{\alpha} \nu \dot{\varepsilon} \varkappa \alpha \lambda \eta \sigma i[\alpha] \nu \delta \varepsilon \varepsilon \lambda \dot{\varepsilon} \chi \theta \eta \dot{v} \pi \dot{\varepsilon} \rho$ 'since Polemarchos, son of Damon, Polemarchos having come upon the assembly, he made a statement about' 


\begin{tabular}{|c|c|c|c|}
\hline No. & References & Date & Formula \\
\hline 26. & $F d D 3.142$ & Hellenistic & 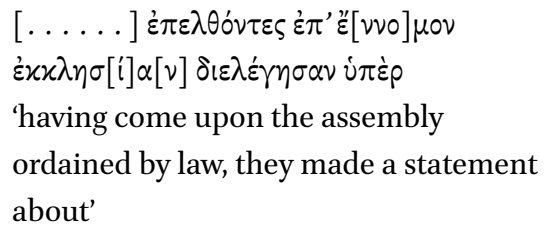 \\
\hline
\end{tabular}

27. $\quad F d D 1.492$

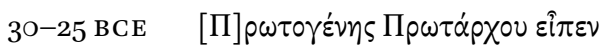

'[P]rotogenes, son of Protarchos said'

28. $F d D 4.442$

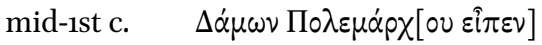

BCE

'Damona, son of Polemarchos said'

29. $F d D 4.443$

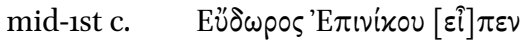

BCE 'Eudoros, son of Epinikos said'

3o. $\quad F d D 4.59$

$1-17 \mathrm{CE}$

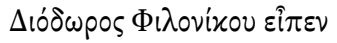

'Diodoros, son of Philonikos said'

31. $\quad F d D 2.102$

$129 \mathrm{CE}$

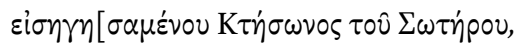

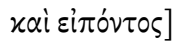

'when Kteson, son of Soteros, proposed

and said'

32. $\quad F d D 2.103$

$129 \mathrm{CE}$

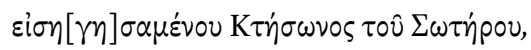

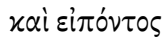

'when Kteson, son of Soteros, proposed

and said' 